NOTE

\title{
Fractal dimension of a coral reef at ecological scales: a discussion
}

\author{
David M. Mark \\ Department of Geography, State University of New York at Buffalo, Amherst, New York 14260, USA
}

\begin{abstract}
In their recent paper, Bradbury and Reichelt (1983) reported and interpreted fractal dimensions for a coral reef. While their data collection technique is innovative and useful, their analysis procedure is incorrect. Their data allow the fractal dimension of the reef they studied to be estimated at 1.13 to 1.16 , not 1.9 to 2 , as the authors reported. Their interpretations of unusually high dimensions are thus unfounded.
\end{abstract}

Recently, Bradbury and Reichelt (1983) applied the fractals model (Mandelbrot, 1977, 1982) to the problem of describing the variations of coral reef complexity with scale. They claimed that coral reefs have fractal dimensions of between 1.9 and 2.0, values which are much higher than those generally reported for coastlines. This in turn implies that the reefs are essentially space-filling, and the authors draw an analogy to other biological structures, such as the human lung. This type of work is to be encouraged, as there have been relatively few actual applications of fractals in the natural sciences. However, the authors' contribution is lessened considerably by the fact that their method for the empirical estimation of fractal dimensions is incorrect. Based on the authors' data, the coral reef they studied actually has a surprisingly low fractal dimension; thus the interesting 'explanations' of the apparently very high dimensions are inappropriate. The difficulties with their methods are explained below.

Fractals are a class of mathematical functions which are continuous and yet not differentiable; they have been described and illustrated by Mandelbrot (1977, 1982). For many fractals, including the ones under consideration here, the functions have the property of statistical self-similarity; that is, the functions are scale-free, and their variation over any interval will be essentially the same, regardless of the length of the interval. For example, if a shoreline were truly a fractal line, then it would not be possible to estimate the realworld length of a reach of shoreline represented by 100 points spaced $1 \mathrm{~mm}$ apart on a piece of paper; whether the line represented a $1 \mathrm{~m}$ or a $100 \mathrm{~km}$ stretch, the appearance of a true fractal would be the same.

Fractals have a number of properties which can be used to estimate their fractal dimensions. One property may be termed the 'dividers relationship'. One wellknown way to measure the length of an irregular line is to set a pair of dividers to some fixed distance $X$, and then to walk the dividers along the line, counting the steps; if there are $N(X)$ steps, then the estimated length of the line is the product $\mathrm{X} \cdot \mathrm{N}(\mathrm{X})$. Anyone who has measured line lengths in this way will know that the result depends on the step size. Generally, if the step size is halved, the number of steps will more than double, and the length will increase. On a continuous, smooth curve, such as the graphic representation of a geographic line, the product $X^{*} N(X)$ will eventually converge toward some limit, the 'true' length of the line. For fractals, however, the length never converges; halving the interval always gives more than twice the number of steps, since more and more of the selfsimilar detail is picked up. For fractals, $N(X)$ plots as a straight line against $X$ on double logarithmic graph paper, with a slope of $-D$, where $1<D<2$ is the fractal dimension of the line. Given $N(X)$ for 2 different values of $X$, the dimension can be estimated (Goodchild, 1980) as:

$$
\mathrm{D}=\log \left(\mathrm{N}\left[\mathrm{X}_{1}\right] / \mathrm{N}\left[\mathrm{X}_{2}\right]\right) / \log \left(\mathrm{X}_{2} / \mathrm{X}_{1}\right)
$$

A second method for estimating the fractal dimension of a phenomenon is based on the variogram of the fractal. For certain fractal functions, the expected squared difference of the value of the function behaves with distance as:

$$
E\left[(W(p)-W(p+t))^{2}\right]=k t^{2 H}
$$

where $\mathrm{H}=2-\mathrm{D}$ for a fractal line function; $\mathrm{H}=3-\mathrm{D}$ for a fractal surface. Here, the fractal dimension can be 
estimated from the slope of the log-log plot of squared function value difference against distance.

Bradbury and Reichelt (1983) discuss only this latter method, and review the principle correctly. They collected data on the number of steps of length $\mathrm{L} / 10$ within individual steps of length $L$, and determined average values over 25 samples for each value of $L$. However, instead of directly applying the dividers relation, they attempted to use the variogram relation. 'The number of these smaller steps that fitted within each larger step is our measure whose variance was used to estimate D' (Bradbury and Reichelt, 1983, p. 170). This variance of the number of steps has no simple relation to a variogram, which plots variance of estimates of some function at point separated by some distance, against that distance. In fact, it is not clear whether the variogram approach can be applied at all for closed curves in the plane, or for segments of such curves; it applies straight-forwardly only to singlevalued functions of one or more coordinates. The fact that a regression of the logarithm of the variance of the number of small steps per larger step, against logarithm of large step size, has a slope of 0.046 does not imply a fractal dimension of near 2 , as the authors suggest.

An example of why the variogram relation cannot be applied here is provided by a geometrically self-similar fractal, the 'triadic Koch island' (Mandelbrot, 1977. p. 36) This construction begins with an equilateral triangle. Then, at each step, each side is replaced by 4 segments, each with a length which is $1 / 3$ of the original side length, and for which the end segments are colinear with the original, while the others replace the middle $1 / 3$ with the other 2 sides of an equilateral triangle. Using the dividers relationship, the fractal dimension is $\mathrm{D}=\log 4 / \log 3$, or 1.2618 . However, every large step of length $\mathrm{L}$ would contain exactly 4 steps of length L/3 (assuming we are 'in phase' with the construction), and hence the variance of the number of steps per interval would be zero. For curves which are not geometrically self-similar, the principle of statistical self-similarity implies that for a fractal, all descriptive statistics will be independent of sampling interval and resolution. For a true fractal curve, both the mean number of steps of length $\mathrm{L} / 10$ within an interval of length $\mathrm{L}$ and the variance about this mean should be independent of the length $L$ over which they are estimated. On a fractal then, the variance of the number of small steps should be constant over all scales, regard- less of the fractal dimension of the curve. In fact, this may be a useful way to confirm that a curve is a fractal. The authors' results (see their Table 1) do not conflict with this hypothesis, and hence serve to support the application of the fractals model coral reefs, but neither do they support their conclusion of an unusually high dimension.

The data in the authors' Table 1 provide a convenient estimate of the fractal dimension of the coral reef they studied: one can apply the dividers relationship outlined above directly to their data on mean number of steps of length $\mathrm{L} / 10$ per step of length $\mathrm{L}$. If logarithms to the base 10 are used, the calculation is especially simple. The denominator of the estimate, $\log \left(\mathrm{X}_{2} / \mathrm{X}_{1}\right)$, is in this case always 1 , and so the estimated dimension is just $\log _{10}(\mathrm{~S})$, where $\mathrm{S}$ is the mean number of sub-steps per step length. The authors' data thus imply a fractal dimension of around 1.13 to 1.16 . This is actually rather smooth, as coastlines go, and the authors' arguments regarding space filling by organic systems are completely unsupported by their data. Summary: The authors' data collection technique, that of counting the number of steps of length $\mathrm{L} / 10$ within each of a sample of length $L$ should prove very useful in field or other manual collection of data to estimate fractal dimensions. In fact, if a 10:1 step length ratio is used, the fractal dimension $D$ is just $\log _{10}(S)$, where $S$ is the mean number of sub-steps. This method of data collection is to be recommended. On the other hand, these and all other authors should be more careful about extending or altering techniques without a more complete understanding of the mathematical models upon which such techniques are often based.

Acknowledgements. I wish to thank Lawrence Kikuchi for bringing this article to my attention.

\section{LITERATURE CITED}

Bradbury, R. H., Reichelt, R. E. (1983). Fractal dimension of a coral reef at ecological scales. Mar Ecol. Prog. Ser. 10: $169-171$

Goodchild, M. F. (1980). Fractals and the accuracy of geographical measures. Math. Geol. 12: 85-98

Mandelbrot, B. B. (1977). Fractals: form, chance, and dimension. San Francisco, Freeman

Mandelbrot, B. B. (1982). The fractal geometry of nature. San Francisco, Freeman

Accepted for printing on May 1, 1983 